\title{
Pattern Formation in Inclined Layer Convection
}

\author{
Karen E. Daniels, Brendan B. Plapp, * and Eberhard Bodenschatz ${ }^{\dagger}$ \\ Laboratory of Atomic and Solid State Physics, Cornell University, Ithaca, New York 14853-2501
}

(Received 21 December 1999)

\begin{abstract}
We report experiments on thermally driven convection in an inclined layer of large aspect ratio in a fluid of Prandtl number $\sigma \approx 1$. We observed a number of new nonlinear, mostly spatiotemporally chaotic, states. At small angles of inclination we found longitudinal rolls, subharmonic oscillations, Busse oscillations, undulation chaos, and crawling rolls. At larger angles, in the vicinity of the transition from buoyancy- to shear-driven instability, we observed drifting transverse rolls, localized bursts, and drifting bimodals. For angles past vertical, when heated from above, we found drifting transverse rolls and switching diamond panes.
\end{abstract}

PACS numbers: 47.54.+r, 05.45.Jn, 47.20.Bp, 47.27.Te

Rayleigh-Bénard convection (RBC) of a horizontal fluid layer heated from below has long served as a paradigm for pattern forming systems [1]. Variations that alter the symmetries, such as Poiseuille flow [2], rotation around a vertical axis [3], and vertical vibrations [4], continue to lead to important insights. Another case, of particular meteorological and oceanographic interest, is RBC of a fluid layer inclined with respect to gravity. This system is not only well suited for the study of buoyancy and shear flow driven instabilities, but may also serve, along with liquid crystal convection [5] and Poiseuille-Bénard convection [2], as a paradigm for anisotropic pattern forming systems.

As with RBC, the onset of inclined layer convection (ILC) occurs when the temperature difference $\Delta T$ across the layer is sufficient for convection rolls to form. The main difference from RBC is that the patternless base state is characterized not only by a linear temperature gradient but also by a symmetry-breaking shear flow. As shown in Fig. 1, the component of gravity tangential to the fluid layer, $\mathbf{g}_{\|}$, causes buoyant fluid to flow up along the warm plate and down along the cold plate. For small angles of inclination $\theta$, buoyancy dominates over shear flow, and the primary instability is to longitudinal rolls (LR) whose axes are aligned with the shear flow direction [6]. With increasing $\theta$, buoyancy effects decrease, and for $\theta>90^{\circ}$ buoyancy is stabilizing. Above a critical angle $\theta_{\mathrm{c}}$ the shear flow causes a primary instability to transverse rolls (TR) with roll axes perpendicular to the shear flow [6]. The few prior experiments [7] on ILC showed reasonable agreement with the linear theory $[6,8-10]$. These experiments also demonstrated that LR are unstable to some form of undulations [7], in qualitative agreement with theory $[9,10]$, but the quantitative details of the state were inaccessible due to experimental limitations.

Here we report the first experimental results on pattern formation in ILC for large aspect-ratio systems in a range of inclination angles $0^{\circ} \leq \theta \leq 120^{\circ}$, i.e., from horizontal to past vertical. We found many unpredicted states when increasing $\Delta T$ above the critical temperature difference, $\Delta T_{\mathrm{c}}$. For $0^{\circ} \leq \theta \leq 77.5^{\circ}$ we observed longitudinal rolls, subharmonic oscillations, Busse oscillations, undula- tion chaos, and crawling rolls. In the neighborhood of the codimension two point [8] for thermal and shear-driven instability $\left(77.5^{\circ} \lesssim \theta \lesssim 84^{\circ}\right.$ ), we observed drifting bimodals, drifting transverse rolls, and localized longitudinal and transverse bursts. For inclinations $\theta \geqslant 84^{\circ}$ we found drifting transverse rolls, switching diamond panes, and longitudinal bursts. Most of these novel states were spatiotemporally chaotic and were found very close to onset, where theoretical progress should be possible.

Experiment. - Our experimental apparatus consisted of a water-cooled pressure chamber containing a convection cell of diameter $10 \mathrm{~cm}$, subdivided into two large aspect ratio rectangular cells. The experimental design was similar to the one described in [11]. The optically flat upper and lower plates of the convection cell consisted of $1 \mathrm{~cm}$ thick single crystal sapphire and single crystal silicon, respectively. The sapphire plate was cooled by a water bath, while the silicon plate was heated by an electric film heater. The convection patterns were visualized by the shadowgraph technique [11]. The sidewalls were constructed of nine layers of notebook paper, providing the best possible thermal matching between cell boundaries and the fluid, reducing sidewall forcing [11]. As measured interferometrically, the plates were parallel to $\pm 0.5 \mu \mathrm{m}$. The pressure chamber housing the convection cell held both the cooling water and the convecting gas to (41.37 \pm $0.01)$ bars, regulated to $\pm 5 \times 10^{-3}$ bar. The temperatures of the two plates were regulated to $\pm 0.0003{ }^{\circ} \mathrm{C}$. Throughout the experiment the mean temperature was kept (a)

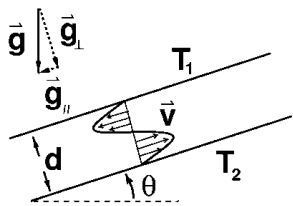

(b)

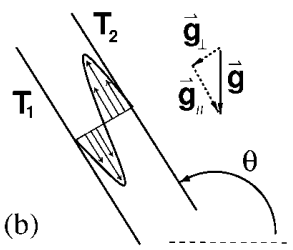

FIG. 1. Schematic of the base flow. (a) Heated from below and (b) heated from above; cell height $d$, gravitational acceleration g, shear flow $\mathbf{v}$, and temperature difference $\Delta T \equiv T_{2}-T_{1}$ with $T_{2}>T_{1}$. 


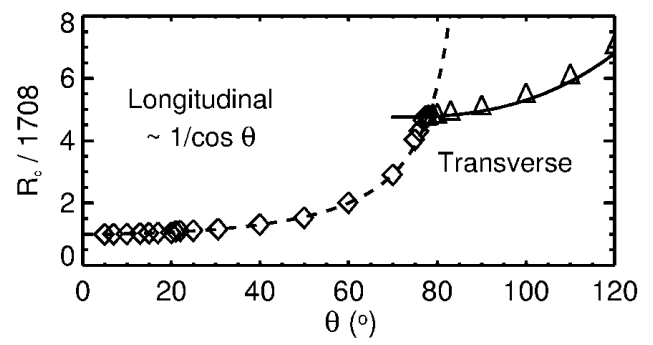

FIG. 2. Onset of longitudinal rolls $(\diamond)$ and drifting transverse rolls $(\triangle)$. Also plotted are the predicted onsets for longitudinal (dashed) and transverse rolls (solid) [13].

constant at $(27.00 \pm 0.05){ }^{\circ} \mathrm{C}$. We determined the cell height $d$ by measuring the pattern wave number at onset for $\theta<60^{\circ}$ and comparing it with the theoretical value of $q_{c}=3.117 / d$. We found $d=(710 \pm 7) \mu \mathrm{m}$ and $d=$ (702 \pm 7$) \mu \mathrm{m}$ for two sets of experiments. The two convection cells had a size $\Gamma_{1} \simeq(21 \times 42) d^{2}$ and $\Gamma_{2} \simeq$ $(14 \times 48) d^{2}$, with the shorter sides oriented perpendicular to the incline. This geometry was chosen to ensure a two-dimensional shear flow profile across the flow [12]. We report quantitative results from cell 1; there was no significant difference in the results for cell 2 . For all data, the Prandtl number was $\sigma \equiv \nu / \kappa=1.07$ as determined in [11], with the kinematic viscosity $\nu$ and thermal diffusivity $\kappa$. The vertical thermal diffusion time was $\tau_{\mathrm{v}} \equiv$ $d^{2} / \kappa=3.0 \mathrm{~s}$. Inclines from $0^{\circ}$ (horizontal) to $120^{\circ}\left(30^{\circ}\right.$ past vertical) were possible, with an accuracy of $\pm 0.02^{\circ}$. Following [1] we calculated the Boussinesq number $\mathcal{P}(\theta)$ for the corresponding horizontal layer to estimate nonBoussinesq effects. At $\Delta T_{\mathrm{c}}(\theta)$ for $\theta<70^{\circ}$ we found $\mathcal{P}(\theta)<1.0$, putting the flow into the Boussinesq regime. For larger angles $\mathcal{P}$ increased linearly to 3.0 for the largest temperature differences investigated.

Onset of convection. - In ILC, the forward bifurcation to LR is predicted to occur at the critical Rayleigh number $R_{\mathrm{c}}(\theta)=R_{\mathrm{c}}^{t}\left(0^{\circ}\right) / \cos \theta$ where $R_{\mathrm{c}}^{t}\left(0^{\circ}\right)=1708=$ $\alpha d^{3} g \Delta T_{\mathrm{c}} / \kappa \nu$ ( $\alpha$ is the thermal expansion coefficient) [6]. The threshold for the forward bifurcation to shear-driven TR at large inclination angle is more complicated, and can be determined only numerically $[6,9]$.

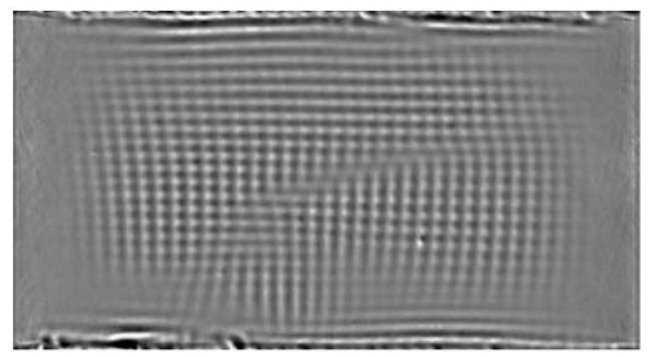

FIG. 3. Digitally enhanced shadowgraph image [14] of bimodals drifting from left to right in cell 1 for $\theta=77.6^{\circ}, \epsilon=$ 0.01 [15]. The rolls at the edges of the cell are caused by sidewall imperfections.
We determined $\Delta T_{\mathrm{c}}$ for convection by quasistatically increasing $\Delta T$ in steps of $1 \mathrm{mK}$ every $20 \mathrm{~min}\left(400 \tau_{\mathrm{v}}\right)$ past the point where convection was observable and then decreasing the temperature difference similarly. For all angles we observed forward bifurcations. Figure 2 shows the measured $R_{\mathrm{c}}(\theta)$, as well as the theoretically predicted onsets for both the longitudinal and the transverse instabilities [13]. We found agreement with theory for the onset of LR: the experimentally observed value was $R_{\mathrm{c}}(\theta)=$ $R_{\mathrm{c}}^{e}\left(0^{\circ}\right) / \cos \theta$ with $R_{\mathrm{c}}^{e}\left(0^{\circ}\right)=1687 \pm 24$. We did not, however, observe the theoretically predicted stationary TR, but instead drifting TR (DTR) at a slightly larger critical Rayleigh number. The drift down the incline may be attributed to the broken symmetry across the layer which is caused by the temperature dependence of the fluid parameters (non-Boussinesq effects). Very interesting is the vicinity of the theoretically predicted codimension two point at $\theta_{\mathrm{c}}=77.76^{\circ}$ [13], where LR and TR have the same onset value. Experimentally, we found a forward bifurcation to DTR above $\theta_{\mathrm{c}}=(77.5 \pm 0.05)^{\circ}$, and in the range $77.5^{\circ} \leq \theta \leq 84^{\circ}$ DTR lost stability to drifting bimodals (DB) above $\epsilon \approx 0.001$. As shown in Fig. 3, DB consist of a superposition of LR and DTR. Here $\epsilon \equiv \frac{\Delta T}{\Delta T_{\mathrm{c}}(\theta)}-1$ is the reduced control parameter. Theoretically, Fujimura and Kelly [8] predicted a forward bifurcation to transverse rolls, which lose stability to bimodals at $\epsilon \approx 0.001$ in a narrow angular region. We find good agreement with these predictions, but with the difference that the experimentally observed patterns are drifting.

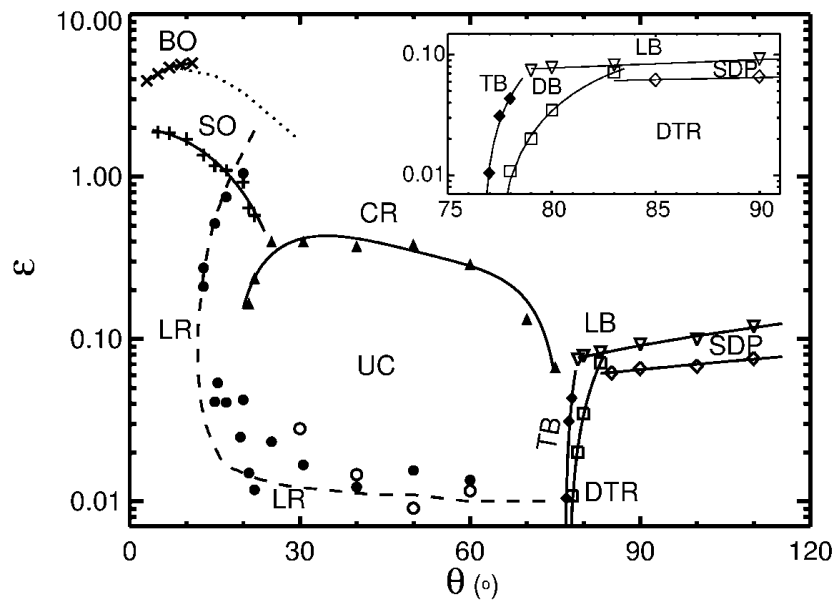

FIG. 4. $(\epsilon, \theta)$ phase space showing the boundaries between the different nonlinear states. LR (longitudinal rolls), BO (Busse oscillations), SO (subharmonic oscillations), UC (undulation chaos), CR (crawling rolls), DTR (drifting transverse rolls), DB (drifting bimodals), LB (longitudinal bursts), TB (transverse bursts), and SDP (switching diamond panes). The dotted line is the predicted onset of Busse oscillations for $\sigma=0.7$ [9], the dashed line is the predicted onset of undulations [13], and the solid lines are guides to the eye. Open circles (UC) were measured via defect density [16], open diamonds (SDP) were measured via correlation length [16], and the remainder were measured visually. The inset shows a magnification of the codimension-two region. 


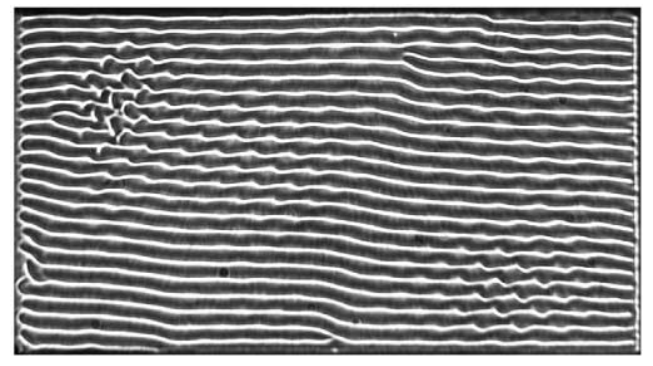

FIG. 5. Contrast enhanced shadowgraph image [14] of subharmonic oscillations in cell 1 , with a turbulent burst in the upper left corner. $\theta=17^{\circ}, \epsilon=1.5$ [15].

Nonlinear states. - Figure 4 shows the measured phase boundaries for the ten observed nonlinear convective states. At low angles $\left(\theta<13^{\circ}\right)$, LR are stable up to $\epsilon \simeq 1$, above which the novel state of subharmonic oscillations (SO) sets in. These oscillations are characterized by a pearl-necklace-like pattern of bright (cold) spots that travel along a standing wave pattern of wavy rolls. As shown in Fig. 5, these oscillations appear in patches whose location changes in time. Typical frequencies of the oscillations were measured to be 1 to 3 cycles per $\tau_{\mathrm{v}}$. A recent theoretical analysis has shown agreement with this value [17]. With further increase in $\epsilon$, localized patches of traveling oscillations burst intermittently. Within $\mathcal{O}\left(\tau_{\mathrm{v}}\right)$ the amplitude of the rolls' waviness increases, the pattern tears transverse to the rolls as shown in the upper left corner of Fig. 5, and fades away leaving an almost parallel roll state.

For $\theta \simeq 10^{\circ}$ and $\epsilon \geq 4$, we observed patches of the well-described Busse oscillations (BO) coexisting with patches of the SO $[1,18]$. As shown by the dotted line in Fig. 4, our data for the onset of the $\mathrm{BO}$ agrees well with

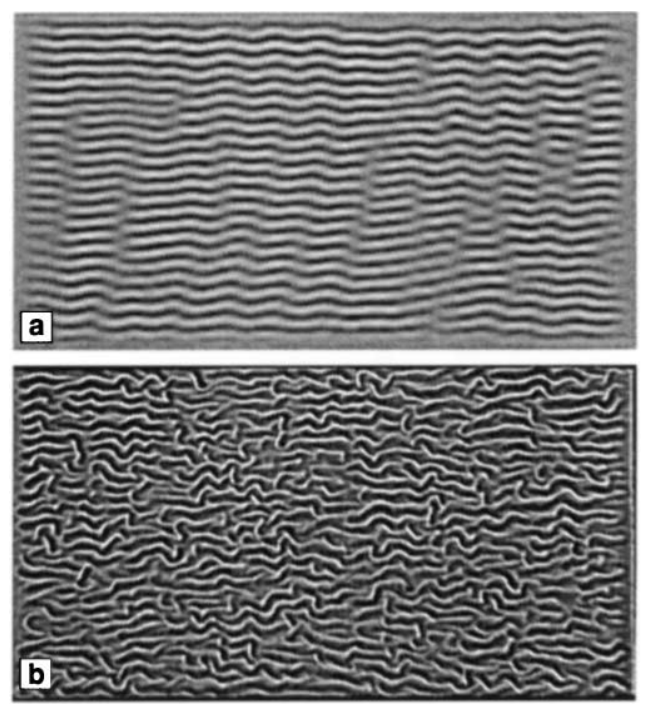

FIG. 6. Digitally enhanced shadowgraph images [14] of convection states at $\theta=40^{\circ}$ in cell 1 . (a) Undulation chaos at $\epsilon=0.07$. (b) Crawling rolls at $\epsilon=0.88$ [15]. the theoretical prediction calculated for $\sigma=0.7$ [9]. It is surprising, however, that both oscillations ( $\mathrm{SO}$ and $\mathrm{BO}$ ) coexist as localized patches in the same cell.

At intermediate angles $\left(25^{\circ}<\theta<70^{\circ}\right)$, where the initial instability is to LR we found with increasing $\epsilon$ that LR were unstable to undulations. Although the experimentally determined value for the instability $\epsilon \approx 0.01$ agrees well with the theoretical prediction (see Fig. 4) $[9,10,13]$, we did not observe a stationary pattern of undulations, but a defect-turbulent state of undulation chaos (UC), similar to that found in electroconvecting nematics [19]. A snapshot of UC is shown in Fig. 6a. At $\epsilon \gtrsim 0.11$, the UC begins to "twitch" in the direction transverse to the rolls on time scales $\mathcal{O}\left(\tau_{\mathrm{v}}\right)$. With increasing $\epsilon$, the amplitude of the twitching increases and the rolls eventually tear, with the ends "crawling" in the direction transverse to the original rolls. A snapshot of crawling rolls (CR) is shown in Fig. $6 b$.

In the vicinity of the codimension-two point, at $\theta_{\mathrm{c}}$, we observed drifting bimodals quite close to onset. As shown in Fig. 4, for small angles the existence region of the pure DB is limited by localized transverse bursts (TB), while for large angles by DTR. The TB appear subharmonically, suggesting a secondary instability [20]. A snapshot of transverse bursts and the evolution of a single burst is shown in Fig. 7. In this region of phase space the LR occur in patches that grow and decay intermittently while TB nucleate in high amplitude LR regions. As shown in the time series in Fig. 7, TB grow over the period of a few $\tau_{\mathrm{v}}$ and then decay rapidly. Above $\epsilon \approx 0.8$ the DB are unstable to localized longitudinal bursts (LB) as shown in Fig. 8a. As shown in Figs. 8b-8i, a few longitudinal rolls grow locally to large amplitude and then quickly fade. With both types of bursts, the bursts increase in both density and frequency when $\epsilon$ is increased, eventually developing into a turbulent state at $\epsilon \geq 1$.

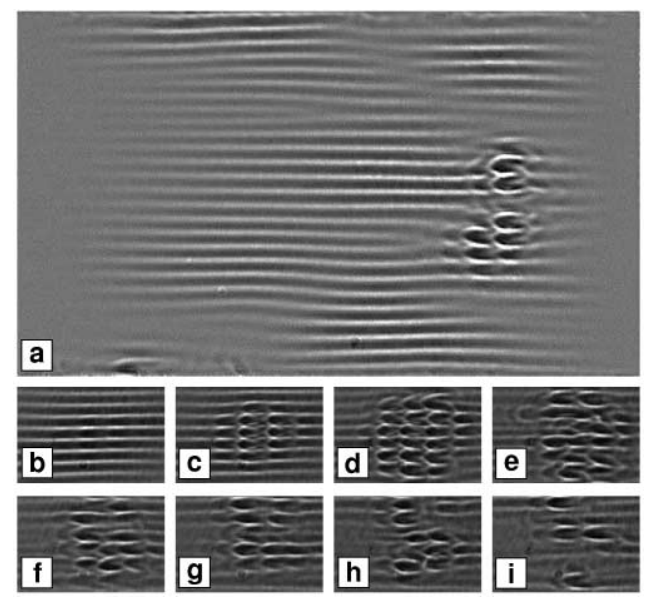

FIG. 7. Digitally enhanced images [14] of (a) transverse bursts in spatiotemporally chaotic longitudinal rolls, at $\theta=77^{\circ}$ and $\epsilon=0.04$ in cell 1 [15]. (b) -(i) Time evolution of a single burst at time intervals $0.36 \tau_{\mathrm{v}}$. 


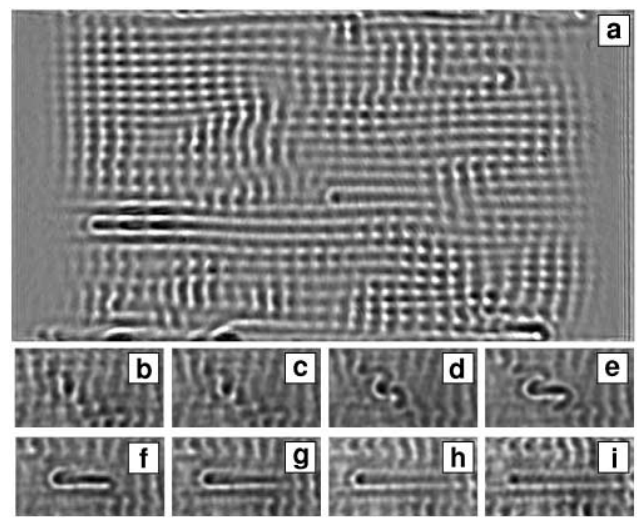

FIG. 8. Digitally enhanced images [14] of (a) longitudinal bursts at $\theta=79^{\circ}$ and $\epsilon=0.10$ in cell 1 [15]. (b)-(i) Time evolution of a single burst at time intervals $0.09 \tau_{\mathrm{v}}$.

Past $90^{\circ}$, we continued to observe shear-driven convection patterns. DTR are the primary instabilities; however, they are unstable to switching diamond panes (SDP) at $\epsilon \approx 0.07$. The state is characterized by spatiotemporally chaotic switching on time scales of $\mathcal{O}\left(\tau_{\mathrm{v}}\right)$ from $+45^{\circ}$ to $-45^{\circ}$ of large amplitude regions of DTR, as seen in Fig. 9a. At $\epsilon \gtrsim 0.1$, SDP are unstable to LB.

Inclined layer convection in the weakly nonlinear regime displays a rich phase diagram, with ten different states accessible over the range of parameters investigated. The phase space naturally divides into several regions of characteristic behavior which have so far been characterized semiquantitatively. All states but LR and DTR are spatiotemporally chaotic. Most instabilities occurred very close to onset and further theoretical description should be possible. Especially interesting is the bursting behavior, which may be related to turbulent bursts in other shear flows [21].

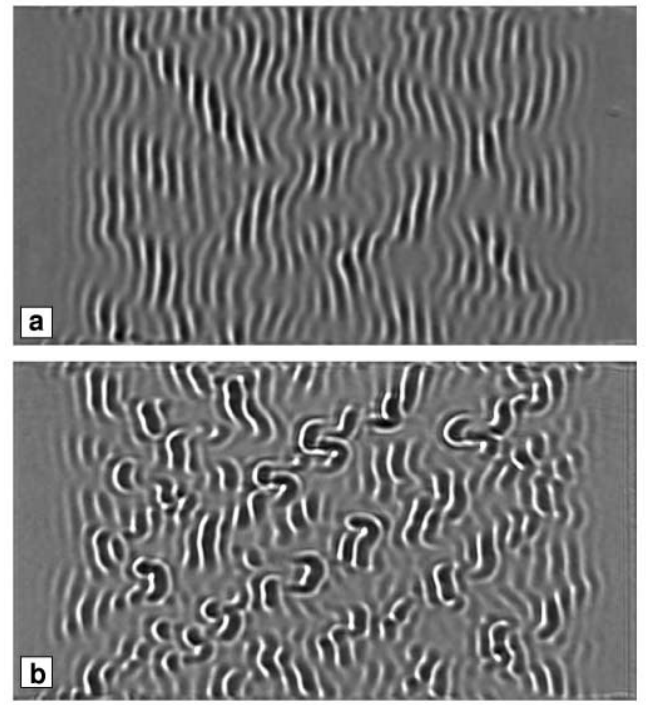

FIG. 9. Digitally enhanced shadowgraph images [14] of (a) switching diamond panes $\left(\epsilon=0.1, \theta=100^{\circ}\right)$ and (b) longitudinal bursts within diamond panes $\left(\epsilon=0.19, \theta=100^{\circ}\right)$ in cell 1 [15].
We thank F. H. Busse and W. Pesch for important discussions on the stability curves and theoretical descriptions of various states. E. B. acknowledges the kind hospitality of H. Levine at the University of California at San Diego, where part of this manuscript was prepared. We gratefully acknowledge support from the NSF under Grant No. DMR-9705410.

*Present address: Center for Nonlinear Dynamics, University of Texas at Austin, Austin, TX 78712.

†Email address: eb22@cornell.edu

[1] E. Bodenschatz, W. Pesch, and G. Ahlers, Annu. Rev. Fluid Mech. 32, 709 (2000), and references therein.

[2] C. H. Yu, M. Y. Chang, and T. F. Lin, Int. J. Heat Mass Transf. 40, 333 (1997), and references therein.

[3] K. M. S. Bajaj, J. Liu, B. Naberhuis, and G. Ahlers, Phys. Rev. Lett. 81, 806 (1998); Y. Hu, W. Pesch, G. Ahlers, and R. E. Ecke, Phys. Rev. E 58, 5821 (1998).

[4] J. L. Rogers, M.F. Schatz, J. L. Bougie, and J. B. Swift, Phys. Rev. Lett. 84, 87 (2000).

[5] L. Kramer and W. Pesch, Annu. Rev. Fluid Mech. 27, 515 (1995).

[6] Y. Chen and A.J. Pearlstein, J. Fluid Mech. 198, 513 (1989), and references therein.

[7] J. E. Hart, J. Fluid Mech. 47, 547 (1971); D. W. Ruth, G. D. Raithby, and K. G. T. Hollands, J. Fluid Mech. 96, 481 (1980); J. N. Shadid and R. J. Goldstein, J. Fluid Mech. 215, 61 (1990), and references therein.

[8] K. Fujimura and R. E. Kelly, J. Fluid Mech. 246, 545 (1993); in Bifurcation Phenomena and Chaos in Thermal Convection, edited by H. H. Bau et al. (ASME, New York, 1992), Vol. 214, p. 73.

[9] R. M. Clever and F.H. Busse, J. Fluid Mech. 81, 107 (1977).

[10] F. H. Busse and R. M. Clever, J. Eng. Math. 26, 1 (1992).

[11] J. R. de Bruyn et al., Rev. Sci. Instrum. 67, 2043 (1996).

[12] B. B. Plapp, Ph.D. thesis, Cornell University, 1997.

[13] W. Pesch (private communication).

[14] See AIP Document No. EPAPS: E-PRLTAO-84-047023 for MPEG movies of spatiotemporally chaotic convection states. This document may be retrieved via the EPAPS homepage (http://www.aip.org/pubservs/epaps.html) or from ftp.aip.org in the directory /epaps/. See the EPAPS homepage for more information.

[15] The left side of the cell is higher than the right, with warm upflow to the left and cold downflow to the right.

[16] K. E. Daniels and E. Bodenschatz (unpublished).

[17] F. H. Busse and R. M. Clever (private communication).

[18] F. H. Busse, J. Fluid Mech. 52, 97 (1972). Please note that the oscillations described in Yu. A. Astrov, I. Müller, E. Ammelt, and H. G. Purwins, Phys. Rev. Lett. 80, 5341 (1998), appear to be an instance of the same phenomenon.

[19] I. Rehberg, S. Rasenat, and V. Steinberg, Phys. Rev. Lett. 62, 756 (1989).

[20] L. Gil (private communication).

[21] E. Knobloch and J. Moehlis, in Pattern Formation in Continuous and Coupled Systems, edited by M. Golubitzki et al. (Springer-Verlag, Berlin, 1999). 